\title{
Uterine EMG Signals Spectral Analysis for Pre-Term Birth Prediction
}

\author{
Markos G. Tsipouras \\ Department of Informatics and Telecommunications Engineering, \\ University of Western Macedonia \\ Kozani, GR47100, Greece \\ mtsipouras@uowm.gr
}

\begin{abstract}
A methodology for prediction of pre-term births is presented in this paper. The methodology is based on the analysis of EHG signals and data mining techniques. Initially, spectral and non-linear characteristics of the EHG are extracted, forming a pattern that is used to train a classifier to discriminate between term and pre-term cases. The method has been tested using a benchmark EHG database, and the obtained results indicate its effectiveness in accurate pre-term/term labour prediction.
\end{abstract}

Keywords-preterm delivery; electrohysterogram; EHG signal processing; uterine electromyogram; prediction

\section{INTRODUCTION}

According to the world health organization (WHO) 15 million babies are delivered before the 37th week of gestation every year, being more than 1 in 10 cases [1]. Premature birth is the leading cause of death in children under 5 years, while premature babies develop lifetime disabilities, including learning disabilities and visual and hearing problems [2]. In addition, premature births create an enormous economic impact on the health care systems, estimated at 3 billion $£$ in England and Wales [3], and continues to be significantly higher for preterm born children in the first 5 [4] and 10 [5] years of life. Thus, developing techniques for premature birth prediction is an important task that have gained attention in the past decades, since accurate prediction of premature labour can lead to their significant limitation. Premature birth prediction has been mainly based on calculating risk factors, largely associated with the medical condition of the mother, including diabetes, hypertension, smoking, abnormalities of the uterus and others [6]. An alternative approach that have gained significant interest is the analysis of the electrohysterogram (EHG), which is the electromyogram (EMG) of the uterus, thus recording the muscular activity of the uterine during gestation. Being noninvasive (recorded on the abdominal surface) and simple to obtain, EHG have proven to be a significant monitoring tool during pregnancy and labour [7].

Moving a step forward, several studies have successfully used EHG signal to forecast pre-term labour [8-14]. These approaches mainly focus on the EHG signal processing, by extracting several time, frequency and/or non-linear characteristics from it, and then applying statistical analysis $[8$, 12] or classification techniques [9-11, 13-14] for pre-term/term cases discrimination. In this study, a methodology for EHG signal analysis for predicting premature births is presented. The methodology is based on the analysis of the EHG signal, focusing on its spectral and non-linear characteristics, while the prediction is made using intelligent techniques. A benchmark EHG database has been used for the development and validation of the methodology, and the obtained results indicate its effectiveness in accurate pre-term labour prediction.

\section{RELATED WORK}

\section{A. Database}

The Term-Pre-term ElectroHysteroGram DataBase (TPEHG DB) [8] is a collection of EHG records (uterine EMG records), obtained at the University Medical Centre Ljubljana from 1997 to 2005 . The records were obtained around the 22nd week of gestation or around the 32nd week of gestation, during regular check-ups. The database contains 300 records from equal number of pregnancies, with 262 records obtained from pregnancies with on-term delivery (pregnancy duration $>37$ weeks) and 38 records obtained from pregnancies which ended prematurely (pregnancy duration $\leq 37$ weeks). Also, 162 records obtained before the 26th week of gestation (being 143 term and 19 pre-term) while 138 obtained during or after the 26th week of gestation (with 119 term and 19 pre-term). For each recording, 4 electrodes were used: E1, placed $3.5 \mathrm{~cm}$ to the left and $3.5 \mathrm{~cm}$ above the navel; E2, placed $3.5 \mathrm{~cm}$ to the right and $3.5 \mathrm{~cm}$ above the navel; E3 placed $3.5 \mathrm{~cm}$ to the right and $3.5 \mathrm{~cm}$ below the navel; E4, placed $3.5 \mathrm{~cm}$ to the left and $3.5 \mathrm{~cm}$ below the navel. The differences in the electrical potentials of the electrodes were recorded, producing 3 channels: S1=E2-E1; S2=E2-E3; S3=E4-E3. The sampling frequency was $20 \mathrm{~Hz}$, with each record having duration of 30 minutes with a 16-bit resolution over a \pm 2.5 millivolts range. An example of EHG recording (all channels) is illustrated in Figure 1.

\section{B. Realted works presented in the literature}

Authors in [8] presented a comparison of various linear and non-linear features extracted from EHG to separate term and pre-term records. Their analysis included filtering of the signals in several different frequency bands, being $0.08-4 \mathrm{~Hz}, 0.3-4$ $\mathrm{Hz}$ and $0.3-3 \mathrm{~Hz}$, and extraction of several features, such as 
root mean square, median frequency of the signal power spectrum, autocorrelation zero-crossing, maximal Lyapunov exponent, correlation dimension and sample entropy. Student's t-test was used to evaluate the features for their ability to separate term and pre-term groups.
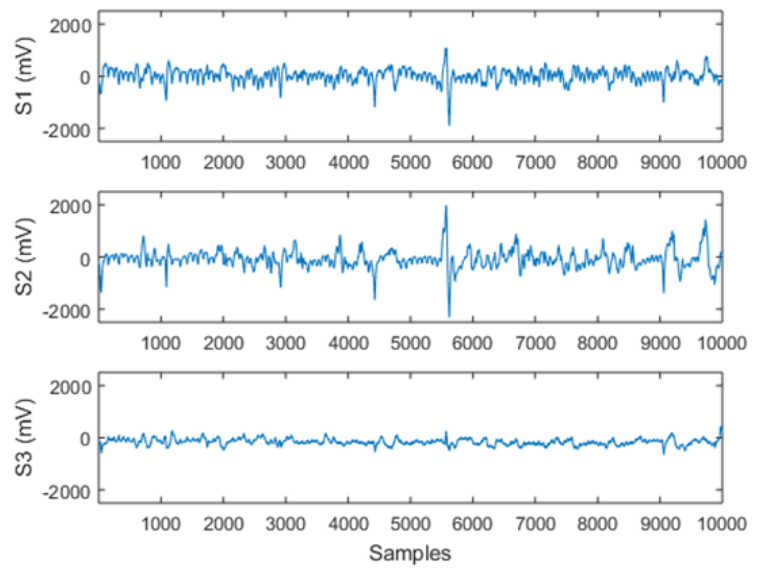

Fig. 1. EHG recording: S1, S2 and S3 chanels.

Authors in [9] focused on the analysis of the channel 3 of the EHG signals, filtered in the $0.34-1 \mathrm{~Hz}$ band. Root mean squares, peak and median frequency, and sample entropy features were extracted from the signals and several different classification algorithms were employed, being linear discriminant (LDA), quadratic discriminant (QDA), uncorrelated normal density, polynomial, logistic, $\mathrm{k}$ nearest neighbours $(\mathrm{kNN})$, decision tree (DT), Parzen and support vector machines (SVM). The dataset was oversampled, using the Synthetic Minority Over-Sampling Technique (SMOTE) to generate 262 pre-term records, using the 38 already available records. Monte Carlo cross-validation (MCCV) $(80 \%$ holdout, 100 iterations) and the $\mathrm{N}$-fold cross validation (with $\mathrm{N}=5$ ) techniques were applied. Authors in [10] employed neural networks (NNs) for the same problem. In this case the signals were filtered in the 0.34 to $1 \mathrm{~Hz}$ band, and root mean square, median frequency, peak frequency and sample entropy features were extracted. The dataset was oversampled using the same technique as in [9] (SMOTE) to generate 262 pre-term signals from the 38 already available. Classification was performed using six different NN classifiers (backpropagation feedforward $\mathrm{NN}$, Levenberg-Marquardt feed-forward $\mathrm{NN}$, automatic $\mathrm{NN}$, radial basis function $\mathrm{NN}$, random $\mathrm{NN}$, perceptron linear classifier). MCCV $(80 \%$ holdout, 30 iterations) and 5-fold cross-validation techniques were employed to validate the obtained results. Following the same methodology of $[9,10]$, authors in [11] analysed channel S3 of the EHG signals, filtered in the $0.34-1 \mathrm{~Hz}$ band, using the same features. Again, several classifiers are tested, including selforganized network Inspired by the immune algorithm (SONIA) and fuzzy SONIA. the pre-term class is oversampled using min and max to produce 262 pre-term records. Cross-validation was performed using $60 \%$ of the data for training, $25 \%$ for validation and $15 \%$ for testing (30 iterations).
Authors in [12] presented a methodology for classification of EHG records using median frequencies of power spectra and sample entropy. In their study, filtering of the signal using several different frequency sub-bands was tested $(0.08-4 \mathrm{~Hz}$; $0.3-4 \mathrm{~Hz} ; 0.3-3 \mathrm{~Hz} ; 0.34-1 \mathrm{~Hz}$ ). The median frequency (frequency value for which the sums of the power above and below it, are equal) of the mean power spectrum, estimated by the adaptive autoregressive (AAR) method with recursive least squares (RLS) algorithm. Also, sample entropy of each signal was calculated. Furthermore, clinical information available for the EHG records (age, parity, abortions, weight, hypertension, diabetes, placental position, bleeding in the first and second trimester, funnelling, smoking) was employed. The tested classifiers included kNN, LDA, QDA, SVM and DT while SMOTE was employed to generate synthetic data so as to balance the two classes. Authors in [13] extracted several time and spectral features from the signal (integrated EMG, mean absolute value, simple square integral value, wavelet length, $\log$ detector, root mean square value, variance, difference absolute standard deviation value, maximum fractal length, average amplitude change, peak frequency, median frequency). Then, feature analysis was performed based on statistical significance, several linear discriminant analysis techniques and Gram-Schmidt analysis, resulting to the four most discriminating features, which were used as input to a classifier. Several artificial NN classifiers were tested, including back-propagation feed-forward NN, LevenbergMarquardt feed-forward NN, perceptron linear classifier, radial basis function $\mathrm{NN}$, random $\mathrm{NN}$, voted perceptron and discriminative restricted Boltzmann machine. As in [9], the dataset was oversampled SMOTE, to generate 262 pre-term records (using the existing 38 records). Cross-validation was applied using the MCCV technique $(80 \%$ holdout, 30 iterations) and the 5-fold technique.

Authors in [14] presented a methodology for prediction of pre-term delivery based on empirical mode decomposition (EMD) combined with wavelet packet decomposition (WPD) of the EHG signals. The signals were filtered with cutoff frequencies of $0.3-3 \mathrm{~Hz}$, and EMD was performed up to 11 levels to obtain the intrinsic mode functions, which were further analysed with 6-level WPD. From the coefficients obtained after the analysis, eight features are extracted (fractal Dimension, fuzzy entropy, interquartile range, mean absolute deviation, mean energy, mean Teager-Kaiser Energy, sample entropy, standard deviation) and particle swarm optimization (PSO) method for feature selection was applied. Adaptive synthetic sampling approach (ADASYN) was applied to increase the number of pre-term signals from 38 to 244 . Several different classifiers were tested, such as linear discriminant analysis, quadratic discriminant, analysis, decision tree, $\mathrm{kNN}$, radial basis, and SVM, while 5-fold and 10-fold cross-validation techniques were employed.

\section{METHODOLOGY}

In this study, the EHG signals are analysed with respect to their spectral characteristics. Initially a set of filters are used to filter all channels of each recording to specific frequency subbands, and then the fractional energy of each frequency subband is calculated. Also, non-linear characteristics are extracted 
from the signals. The extracted features are used to train a random forest classifier, to discriminate term and pre-term recordings according to their spectral behaviour. The flowchart of the proposed methodology is presented in Figure 2.

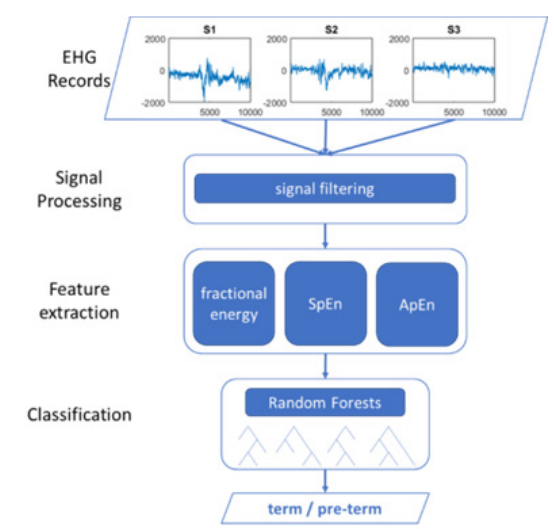

Fig. 2. Flowchart of the proposed methodology.

\section{A. EHG records}

The EHG records used in this study are obtained from the TPEHG DB. All available records (300) are included in the study, and from each record, all three channels (S1, S2 and S3) are analysed. The unfiltered signals are used.

\section{B. Signal Processing}

Initially each channel is filtered with a high pass filter with cut-off frequency of $0.3 \mathrm{~Hz}$ and subsequently with a low pass filter with cut-off frequency of $4 \mathrm{~Hz}$. Then, to access the spectral characteristics of each EHG record, a set of filters are used to filter each channel to a specific frequency sub-band. Several researchers have reported that the uterine electrical activity occurs in the $0 \mathrm{~Hz}-5 \mathrm{~Hz}$ frequency band [8], with the majority laying at the $<1 \mathrm{~Hz}$ spectrum [9]. Furthermore, main respiration rates are $<0.33 \mathrm{~Hz}$. Based on the above, the frequency sub-bands used for this study are: $0.33 \mathrm{~Hz}-0.65 \mathrm{~Hz}$, $0.65 \mathrm{~Hz}-1 \mathrm{~Hz}, 1 \mathrm{~Hz}-2 \mathrm{~Hz}, 2 \mathrm{~Hz}-3 \mathrm{~Hz}$ and $3 \mathrm{~Hz}-4 \mathrm{~Hz}$, selected so as: (i) to exclude respiration rates, (ii) to additionally examine the energy distribution over the $0.33 \mathrm{~Hz}-1 \mathrm{~Hz}$ frequency band, and (iii) to include information from frequency bands $>1 \mathrm{~Hz}$. The $0.33 \mathrm{~Hz}-1 \mathrm{~Hz}$ band has been the sole focus of several studies [9-11, 13-14], however an attempt to look into this band with greater detail (i.e. by examining sub-bands) has never been attempted in the literature before.

\section{Features Extraction}

\section{1) Fractional Energy}

After filtering all channels in the specific frequency subbands, the energy of each of the filtered signals for all channels, is calculated. Based on the energy values, the fractional energy of each frequency sub-bad is calculated:

$$
\text { frac_energy } y_{s b}^{c}=\frac{\operatorname{energy}_{s b}^{c}}{\sum \text { energy } y_{s b}^{c}}
$$

where $c$ is the channel (with $c=\{S 1, S 2, S 3\}$ ), $s b$ is the frequency sub-band (with $s b=\{[0.3 \mathrm{~Hz}-0.65 \mathrm{~Hz}]$,
$[0.65 \mathrm{~Hz}-1 \mathrm{~Hz}],[1 \mathrm{~Hz}-2 \mathrm{~Hz}],[2 \mathrm{~Hz}-3 \mathrm{~Hz}],[3 \mathrm{~Hz}-4 \mathrm{~Hz}]\})$, energy $y_{s b}^{c}$ is the energy of the channel $c$ after filtered in the $s b$ frequency sub-band and frac_energy $y_{s b}^{c}$ is the fractional energy of the channel $c$ after filtered in the $s b$ sub-band.

\section{2) Spectral Entropy}

Spectral entropy (SpEn) is defined as the Shannon entropy of the power spectrum density. Thus, it is calculated for each channel of the EHG signal as follows:

$S p E n^{c}=\frac{-\sum\left(\text { frac_energy }_{s b}^{c} \cdot \log \left(\text { frac_energy }_{s b}^{c}\right)\right)}{\log M}$

$S p E n^{c}$ is the spectral entropy of the channel $c$ and $M$ is the number of frequency sub-bands $(M=5)$.

\section{3) Approximate Entropy}

Approximate Entropy (ApEn) can detect changes in the underlying signal behaviour, which are not directly related to peaks or amplitude variations [15]. $A p E n_{s b}^{c}$ is calculated for each sub-band $s b$ of each channel $c$ :

$$
\begin{gathered}
A p E n_{s b}^{c}=\frac{\sum_{i=1}^{N-m+1} \log C_{i}^{m}(r)}{N-m+1}-\frac{\sum_{i=1}^{N-m} \log C_{i}^{m+1}(r)}{N-m} \\
C_{i}^{m}(r)=\frac{\sum_{j=1}^{N-m+1} \Theta\left(r-\left\|x_{i}-x_{j}\right\|\right)}{N-m+1}
\end{gathered}
$$

where $x$ is the EHG signal, $\Theta(\cdot)$ is the Heaviside step function, $\|\cdot\|$ is the Euclidian norm and $r$ and $m$ are parameters defined as $r=0.15$ and $m=2$ chosen based on the results of previous studies [15] for good statistical validity of ApEn.

\section{4) Features Vector}

For each channel, five fractional energies, spectral entropy and five ApEn values are calculated. Furthermore, a binary feature representing the week of recording is used $(W)$, being 0 for records obtained before the 26th week of gestation and 1 for records obtained during or after the 26th week of gestation. Thus, the feature vector for each record includes 12 features for a single channel, and 34 features for all channels.

\section{Classification}

The database contains 300 records, with 262 term and 38 pre-term records. Since this unbalance between the number of data in the two classes has a major impact in classification algorithms, all researchers employing classification have used data balancing techniques (SMOTE, ADASYN, min\&max), mainly by creating artificial data from the existing to boost the number of data in the minority class. To address this issue in this study (i) the number of pre-term data is increased by repeating all pre-term data twice, and thus the final number of data used in the classification is 262 term and 76 pre-term, and (ii) an appropriate classifier is selected.

Classification is performed using the Random Forest (RF) classifier [16], which is an ensemble learning technique based on the construction of multiple DTs using sub-sets of the initial dataset. RF is constructed with 100 DTs. The selection of this classifier is based on the basic characteristic of the dataset, i.e. being unbalanced, since RF have been reported to appropriate for handling unbalanced datasets, against many other well- 
known classifiers (such as neural networks) [17]. Furthermore, RF performs two feature selection steps, an initial random selection in the feature bagging step and a subsequent selection of the feature with the highest normalized information gain for each tree node, thus it can cope with the large number of extracted features (34 when all channels are used) without the need of an additional feature selection technique. The 10-fold stratified cross-validation technique has been employed in the classification process of this study. Thus, the dataset has been divided into 10 equally sized subsets, and then nine of them are used to train the classifier, and the final for test. Special care has been made so as not to have data from the same subject into both training and test phases. Thus, both records of preterm data (since each pre-term record is repeated twice), are included in the same fold.

\section{RESULTS}

In order to assess the spectral characteristics of each channel of the EHG signals, the features from each channel are initially used individually to predict the term/pre-term pregnancy outcome. The obtained results are in term of sensitivity, positive predictive value (PPV) and classification accuracy, and they are presented in Table I. Individual channel S3 obtained the best results, however the employment of features from all 3 channel has beneficial effect on the classification results.

TABLE I. RESULTS

\begin{tabular}{|c|c|c|c|c|}
\hline \multirow{2}{*}{ Channel } & Class & Sensitivity (\%) & PPV (\%) & $\begin{array}{c}\text { Classification } \\
\text { Accuracy (\%) }\end{array}$ \\
\hline \multirow{2}{*}{ S1 } & Term & $95.00 \%$ & $98.80 \%$ & \multirow{2}{*}{$95.24 \%$} \\
\cline { 2 - 4 } & Pre-term & $96.05 \%$ & $84.88 \%$ & \\
\hline \multirow{2}{*}{ S2 } & Term & $95.04 \%$ & $99.20 \%$ & \multirow{2}{*}{$95.56 \%$} \\
\cline { 2 - 4 } & Pre-term & $97.37 \%$ & $85.06 \%$ & \multirow{2}{*}{$96.15 \%$} \\
\hline \multirow{2}{*}{ S3 } & Term & $95.80 \%$ & $99.21 \%$ & \multirow{2}{*}{$97.93 \%$} \\
\cline { 2 - 4 } & Pre-term & $97.37 \%$ & $87.06 \%$ & $99.23 \%$ \\
\hline \multirow{2}{*}{ all } & Term & $98.09 \%$ & $93.67 \%$ & \multirow{2}{*}{} \\
\cline { 2 - 4 } & Pre-term & $97.37 \%$ & \multicolumn{2}{|c}{} \\
\hline
\end{tabular}

\section{DISCUSSION}

In the proposed methodology for EHG analysis and term/pre-term classification, a comparison among the results obtained from the features extracted from each channel separately and their combination, is presented. The obtained results indicate that analysing features extracted from all channels (S1, S2 and S3) has a positive impact; although results for term class PPV are almost unaffected and the same applies for the pre-term class sensitivity, the sensitivity of the term class increased by more than $2 \%$, while the PPV result for the pre-term class increased by more than $6 \%$. The same applies for the classification accuracy results, where the value for features from all 3 channels is $97.93 \%$, with the best obtained result for an individual channel being $96.15 \%$ (obtained for channel S3). Most of the works presented in the literature are based on the analysis of only the S3 channel [9$11,13]$, since it has been reported to be the most informative [8]. This has been confirmed with the findings of this study, since channel S3 produced the best results among individual channel analysis.
However, the employment of features from all three channels in the analysis lead to significantly more accurate prediction of pre-term births; this has not been reported in the literature before. Furthermore, the $0.33 \mathrm{~Hz}-1 \mathrm{~Hz}$ frequency sub-band has been the focus of several studies [9-11, 13-14], without however attempting to examine the energy distribution within this band. Also, frequency bands $>1 \mathrm{~Hz}$ have been excluded from these studies, although there has been evidence that they contain important information [12]. In this work the frequency sub-bands have been selected so as to lift both of these limitations by: (i) examine in greater detail the most informative sub-band $(0.33 \mathrm{~Hz}-1 \mathrm{~Hz})$, and (ii) include several frequency sub-bands $>1 \mathrm{~Hz}$ in order to access information in higher frequencies. A comparative study of similar methods presented in the literature is presented in Table II.

All methods have been developed and tested using the same database, being the TPEHG DB, however having different classification datasets, since different data balancing techniques have been employed. Most researches employed classification techniques [9-11, 13-14] while also statistical analysis of the extracted features has also been presented. The proposed methodology compares well with other approaches presented in the literature, obtaining $97.4 \%$ sensitivity, $98.1 \%$ specificity, 93.7\% PPV, $99.2 \%$ negative predictive value (NPV) and $97.9 \%$ classification accuracy. These results are the best reported so far, improving the best previously reported sensitivity [14] by $2.3 \%$ and the respective classification accuracy by $1.6 \%$. The differences are higher with other previously reported results (up to $8.4 \%$ for sensitivity, $19.1 \%$ for specificity, $3.7 \%$ for PPV, $9.2 \%$ for NPV and $10.9 \%$ for classification accuracy). However, it should be noted that although the TPEHG DB was employed in all cases, the classification datasets significantly differ since large number of synthetic data are generated in several cases [9-14], while the size of the classification dataset also varies. The issue of balance in the dataset has been reported by all researchers employing classification techniques [9-11, 13-14], with data balancing techniques being employed in all cases. SMOTE has been employed in several works [910, 12-13] to generate additional pre-term data; 262 pre-term cases are generated from the 38 already available. Min\&max was employed in [12] to produce 262 pre-term records, while in [14] ADASYN was applied to increase the number of preterm signals from 38 to 244 . In both cases, the number of generated data is many times larger than the number of existing data (for the pre-term class), thus significantly disturbing the term/pre-term ratio in the initial dataset, which is $12.67 \%$ preterm and $87.33 \%$ term, closely related to the actual reported percentage [1]. Furthermore, the obtained results regarding the pre-term class are mainly (or solely) based in synthetic data.

In this study, no synthetic data are generated or employed in the classification. Instead, to address the unbalanced dataset issue, all pre-term data are included twice in the dataset (resulting to 76 pre-term records). Thus, there are no artificial generated data and the obtained results concern only real cases. Also, the term/pre-term ratio has been kept to values close to the actual recorded ratio $(22.48 \%$ pre-term and $77.51 \%$ term records in the classification dataset). Furthermore, the folds selection has been made so as this repetition not to disturb the validation procedure by having the same record into both 
training and test phases; each pair of repeated pre-term data is included in a single fold.

TABLE II. COMPARATIVE STUDY OF METHODS PRESENTED IN THE LITERATURE, USING THE TPEHG DB

\begin{tabular}{|c|c|c|c|c|c|c|}
\hline Reference & $\begin{array}{l}\text { Channel/ } \\
\text { frequency }\end{array}$ & Analysis/Features & Analysis/ Classification & Data Balancing & $\begin{array}{c}\text { Results } \\
\text { Validation }\end{array}$ & Results \\
\hline$[8]$ & $\begin{array}{c}\text { All / } \\
0.08-4 \mathrm{~Hz} \\
0.3-4 \mathrm{~Hz} \\
0.3-3 \mathrm{~Hz}\end{array}$ & $\begin{array}{l}\text { Linear and non-linear/ } \\
\text { root mean square, median } \\
\text { frequency of the signal power } \\
\text { spectrum, autocorrelation zero- } \\
\text { crossing, maximal Lyapunov } \\
\text { exponent, correlation } \\
\text { dimension and sample entropy. }\end{array}$ & Student's t-test & & & \\
\hline [9] & $\begin{array}{c}\text { Ch. } 3 \text { / } \\
0.34-1 \mathrm{~Hz}\end{array}$ & $\begin{array}{l}\text { Root mean square, median } \\
\text { frequency, peak frequency, sample } \\
\text { entropy }\end{array}$ & $\begin{array}{c}9 \text { classifiers/ } \\
\text { LDA, QDA, uncorrelated } \\
\text { normal density, polynomial, } \\
\text { logistic, kNN, DT, Parzen, } \\
\text { SVM }\end{array}$ & $\begin{array}{c}\text { SMOTE } \\
\text { (generated } 262 \\
\text { pre-term records) }\end{array}$ & $\begin{array}{c}\text { MCCV } \\
\text { (80\% holdout, } \\
100 \text { iterations) } \\
\text { 5-fold CV }\end{array}$ & $\begin{array}{l}\text { Sensitivity: } 96.7 \% \\
\text { Specificity: } 90.0 \%\end{array}$ \\
\hline$[10]$ & $\begin{array}{c}\text { Ch. } 3 / \\
0.34-1 \mathrm{~Hz}\end{array}$ & $\begin{array}{l}\text { Root mean square, median } \\
\text { frequency, peak frequency, sample } \\
\text { entropy }\end{array}$ & $\begin{array}{c}\text { 6 NN classifiers/ } \\
\text { backpropagation feed- } \\
\text { forward NN, Levenberg- } \\
\text { marquardt feed-forward NN, } \\
\text { automatic NN, radial basis } \\
\text { function NN, random NN, } \\
\text { perceptron linear classifier }\end{array}$ & $\begin{array}{c}\text { SMOTE } \\
\text { (generated } 262 \\
\text { pre-term records) }\end{array}$ & $\begin{array}{c}\text { MCCV } \\
\text { (80\% holdout, } \\
30 \text { iterations) } \\
\text { 5-fold CV }\end{array}$ & $\begin{array}{l}\text { Sensitivity: } 96.1 \% \\
\text { Specificity: } 91.9 \%\end{array}$ \\
\hline$[11]$ & $\begin{array}{c}\text { Ch. } 3 / \\
0.34-1 \mathrm{~Hz}\end{array}$ & $\begin{array}{l}\text { Root mean square, median } \\
\text { frequency, peak frequency, sample } \\
\text { entropy }\end{array}$ & $\begin{array}{c}7 \text { classifiers/ } \\
\text { SONIA network. MLP, } \\
\text { Fuzzy-SONIA, kNN, } \\
\text { Dynamic Self- } \\
\text { organising Multilayer } \\
\text { network, decision tree, SVM }\end{array}$ & $\begin{array}{l}\text { Min \& Max } \\
\text { (produce } 262 \text { pre- } \\
\text { term records) }\end{array}$ & $\begin{array}{c}\text { Training: } 60 \% \text {, } \\
\text { Validation: } \\
25 \% \text {, Testing: } \\
15 \% \\
\text { (30 iterations) }\end{array}$ & $\begin{array}{l}\text { Sensitivity: } 89 \% \\
\text { Specificity: } 91 \% \\
\text { PPV: } 90 \% \\
\text { NPV: } 90 \% \\
\text { Accuracy: } 90 \%\end{array}$ \\
\hline$[12]$ & $\begin{array}{c}\text { All / } \\
0.34-1 \mathrm{~Hz} \\
0.3-4 \mathrm{~Hz}\end{array}$ & $\begin{array}{l}\text { AAR/ median frequency, sample } \\
\text { entropy, clinical information (age, } \\
\text { parity, abortions, weight, } \\
\text { hypertension, diabetes, placental } \\
\text { position, bleeding in the first and } \\
\text { second trimester, funnelling, } \\
\text { smoking) }\end{array}$ & $\begin{array}{l}5 \text { classifiers/ } \\
\text { kNN, LDA, QDA, } \\
\text { SVM and DT }\end{array}$ & SMOTE & & $\begin{array}{l}\text { Sensitivity: } 96 \% \\
\text { Specificity: } 79 \% \\
\text { Accuracy: } 87 \%\end{array}$ \\
\hline$[13]$ & $\begin{array}{c}\text { Ch. } 3 / \\
0.34-1 \mathrm{~Hz}\end{array}$ & $\begin{array}{l}\text { Integrated EMG, mean absolute } \\
\text { value, simple square integral value, } \\
\text { wavelet length, log detector, root } \\
\text { mean square value, variance, } \\
\text { difference absolute standard } \\
\text { deviation value, maximum fractal } \\
\text { length, average amplitude change, } \\
\text { peak frequency, median frequency }\end{array}$ & $\begin{array}{c}7 \mathrm{NN} \text { classifiers/ } \\
\text { back-propagation feed- } \\
\text { forward NN, Levenberg- } \\
\text { Marquardt feed-forward NN, } \\
\text { perceptron linear classifier, } \\
\text { radial basis function NN, } \\
\text { random NN, voted } \\
\text { perceptron, discriminative } \\
\text { restricted Boltzmann machine }\end{array}$ & $\begin{array}{c}\text { SMOTE } \\
\text { (generated } 262 \\
\text { pre-term records) }\end{array}$ & $\begin{array}{c}\text { MCCV } \\
\text { (80\% holdout, } \\
30 \text { iterations) } \\
\text { 5-fold CV }\end{array}$ & $\begin{array}{l}\text { Sensitivity: } 91 \% \text {, } \\
\text { Specificity: } 84 \%\end{array}$ \\
\hline$[14]$ & $\begin{array}{l}\text { Ch. } 3 \text { / } \\
0.3-1 \mathrm{~Hz}\end{array}$ & $\begin{array}{c}\text { EMD (11 levels) \& WPD (6 levels) / } \\
\text { fractal Dimension, fuzzy entropy, } \\
\text { interquartile range, mean absolute } \\
\text { deviation, mean energy, mean } \\
\text { Teager-Kaiser Energy, sample } \\
\text { entropy, standard deviation }\end{array}$ & $\begin{array}{c}6 \text { classifiers/ } \\
\text { linear discriminant } \\
\text { analysis, quadratic } \\
\text { discriminant analysis, } \\
\text { decision tree, kNN, radial } \\
\text { basis functions, SVM } \\
\end{array}$ & $\begin{array}{c}\text { ADASYN } \\
\text { (increase pre-term } \\
\text { signals from } 38 \text { to } \\
\text { 244) }\end{array}$ & $\begin{array}{l}\text { 5-fold and 10- } \\
\text { fold CV }\end{array}$ & $\begin{array}{l}\text { Sensitivity: } 95.1 \% \\
\text { Specificity: } 97.3 \% \\
\text { Accuracy: } 96.3 \%\end{array}$ \\
\hline this study & $\begin{array}{c}\text { All / } \\
0.33-5 \mathrm{~Hz}\end{array}$ & Fractional energies, SpEn, ApEn & Random Forests & $\begin{array}{c}\text { Resampling } \\
\text { (increase pre-term } \\
\text { signals from } 38 \text { to } \\
76 \text { ) }\end{array}$ & 10-fold CV & $\begin{array}{l}\text { Sensitivity: } 97.4 \% \\
\text { Specificity: } 98.1 \% \\
\text { PPV: } 93.7 \% \\
\text { NPV: } 99.2 \% \\
\text { Accuracy: } 97.9 \%\end{array}$ \\
\hline
\end{tabular}

Most of the studies presented in the literature experimented with several classification algorithms to identify the most appropriate. In this study RF classifier has been selected, since it has been reported to be able to deal with unbalanced datasets [17]. To validate this selection, several well-known classifiers are additionally tested, including $\mathrm{kNN}$, neural networks, SVM, NNs and deep NNs. All alternative classification algorithms presented significantly lower results, with the neural approaches (both classical and deep) constantly classifying samples biased to the larger (term) class.

\section{CONCLUSIONS}

A methodology for premature birth prediction, based on EHG analysis, is presented in this work. The methodology has been evaluated using a publicly available database, and the obtained results indicate its ability for accurate pre-term labour prediction. The results clearly demonstrate that using 
information from all 3 recorded channels (instead of only one, which is common practice in the literature) and assessing frequency sub-bands $>1 \mathrm{~Hz}$ (usually not included in the analysis in the literature) leads to improved classification accuracy. Furthermore, the combination of spectral and non-linear characteristics carries sufficient information for this task. During the validation procedure, only real data were used (no synthetic recordings were included), while the term/pre-term data ratio was kept close to the one reported from WHO, thus the obtained results are more robust. The methodology achieved higher sensitivity and classification accuracy than the best presented in the literature so far. Future work will mainly focus on the application of the methodology on different data, so as to validate its findings into larger and multicentre datasets.

\section{REFERENCES}

[1] March of Dimes, PMNCH, Save the Children, WHO. Born Too Soon: The Global Action Report on Preterm Birth. Eds CP Howson, MV Kinney, JE Lawn. World Health Organization. Geneva, 2012

[2] L. Liu, S. Oza, D. Hogan, Y. Chu, J. Perin, J. Zhu, J.E. Lawn, S. Cousens, C. Mathers, R.E. Black, "Global, regional, and national causes of under-5 mortality in 2000-15: an updated systematic analysis with implications for the Sustainable Development Goals", Lancet, Vol. 388, pp. 3027-35, 2016

[3] L. J. Mangham, S. Petrou, L. W. Doyle, E. S. Draper, N. Marlow, "The cost of preterm birth throughout childhood in England and Wales", Pediatrics, Vol. 123, No. 2, pp. e312-e327, 2009

[4] S. Petrou, Z. Mehta, C. Hockley, P. Cook-Mozaffari, J. Henderson, M. Goldacre, "The impact of preterm birth on hospital inpatient admissions and costs during the first 5 years of life", Pediatrics, Vol. 112, No. 6, pp. 1290-7, 2003

[5] S. Petrou, "The economic consequences of preterm birth duringthe first 10 years of life", BJOG: an International Journal of Obstetrics and Gynaecology, Vol. 112, No. S1, pp. 10-15, 2005

[6] J. D. Iams, "Prediction and early detection of preterm labor", The American College of Obstetricians and Gynecologists, Vol. 101, No. 2, pp. 402-412, 2003

[7] C. Buhimschi, M. Boyle, R. Garfield, "Electrical activity of the human uterus during pregnancy as recorded from the abdominal surface", Obstetrics \& Gynecology, Vol. 90, pp. 102-111, 1997

[8] G. Fele-Zorz, G. Kavsek, Z. Novak-Antolic, F. Jager, “A comparison of various linear and non-linear signal processing techniques to separate uterine EMG records of term and pre-term delivery groups", Medical \& Biological Engineering \& Computing, Vol. 46, pp. 911-922, 2008

[9] P. Fergus, P. Cheung, A. Hussain, D. Al-Jumeily, C. Dobbins, S. Iram, "Prediction of preterm deliveries from ehg signals using machine learning", PLOS ONE, Vol. 8, No. 10, Art. No. e77154, 2013

[10] I. O. Idowu, P. Fergus, A. Hussain, C. Dobbins, H. Al-Askar, “Advance artificial neural network classification techniques using EHG for detecting preterm births", 8th International Conference on Complex, Intelligent and Software Intensive Systems, Birmingham City University, Birmingham, UK, July 2-4, 2014

[11] A. J. Hussain, P. Fergus, H. Al-Askar, D. Al-Jumeily, F. Jager, "Dynamic neural network architecture inspired by the immune algorithm to predict preterm deliveries in pregnant women", Neurocomputing, Vol. 151, pp. 963-974, 2015

[12] A. Smrdel, F. Jager, "Separating sets of term and pre-term uterine EMG records”, Physiological Measurement, Vol. 36, pp. 341-355, 2015

[13] P. Fergus, I. Idowu, A. Hussain, C. Dobbins, "Advanced artificial neural network classification for detecting preterm births using EHG records", Neurocomputing, Vol. 188, pp. 42-49, 2016

[14] U. R. Acharya, V. K. Sudarshan, S. Q. Rong, Z. Tan, C. M. Lim, J. E. W. Koh, S. Nayak, S. V. Bhandary, "Automated detection of premature delivery using empirical mode and wavelet packet decomposition techniques with uterine electromyogram signals", Computers in Biology and Medicine, Vol. 85, pp. 33-42, 2017

[15] S. M. Pincus, A. L. Goldberger, "Physiological time-series analysis: what does regularity quantify?", American Journal of Physiology, Vol 266, pp. H1643-H1656, 1994

[16] L. Breiman, "Random forests", Machine Learning, Vol. 45, No. 1, pp. 5-32, 2001

[17] I. Brown, C. Mues, "An experimental comparison of classification algorithms for imbalanced credit scoring data sets", Expert Systems with Applications, Vol. 39, pp. 3446-3453, 2012

\section{AUTHOR PROFILE}

Markos G. Tsipouras was born in Athens, Greece, in 1977. He received the diploma degree in Computer Science from the University of Ioannina, Greece, in 1999, and M.Sc. and Ph.D degrees in computer science, in 2002 and 2008 respectively, from the same department. He has participated in several European and National R\&D projects as a researcher/developer and he has published 100 papers in peer-reviewed scientific journals and conference proceedings. Also, he has published 7 book chapters, and he has co-authored one book. His research interests include digital signal and image processing, medical informatics, artificial intelligence, fuzzy logic, data mining, decision support systems and expert systems. 